\title{
SPECTRALLY-RESOLVED RAMAN LIDAR TO MEASURE ATMOSPHERIC THREE-PHASE WATER SIMULTANEOUSLY
}

\author{
Fuchao Liu ${ }^{1,2, *}$, Fan $\mathrm{Yi}^{1,2}$ \\ ${ }^{1}$ School of Electronic Information, Wuhan University, Wuhan 430072, China \\ ${ }^{2}$ State Key Laboratory of Information Engineering in Surveying, Mapping and Remote Sensing, Wuhan \\ University, Wuhan 430079, China \\ *Email:lfc@whu.edu.cn
}

\begin{abstract}
We report on a spectrally-resolved Raman lidar that can simultaneously profile backscattered Raman spectrum signals from water vapor, water droplets and ice crystals as well as aerosol fluorescence in the atmosphere. The lidar emits a 354.8-nm ultraviolet laser radiation and samples echo signals in the $393.0-424.0 \mathrm{~nm}$ wavelength range with a $1.0-\mathrm{nm}$ spectral resolution. A spectra decomposition method is developed to retrieve fluorescence spectra, water vapor Raman spectra and condensed (liquid and/or ice) water Raman spectra successively. Based on 8 different clearsky nighttime measurement results, the entire atmospheric water vapor Raman spectra are for the first time obtained by lidar. The measured normalized water vapor Raman spectra are nearly invariant and can serve as background reference for atmospheric water phase state identification under various weather conditions. For an ice virga event, it's found the extracted condensed water Raman spectra are highly similar in shape to theoretical ice water Raman spectra reported by Slusher and Derr (1975). In conclusion, the lidar provides an effective way to measure three-phase water simultaneously in the atmosphere and to study of cloud microphysics as well as interaction between aerosols and clouds.
\end{abstract}

\section{INTRODUCTION}

The traditional water vapor Raman lidar that extracts the Q-branch vi-rotational Raman signals from atmospheric water vapor using narrowband optical devices works well under clear weather conditions. However, it fails in cloud area: since liquid and ice water Raman spectra overlap in spectrum with that of water vapor, Raman signals from cloud condensed (liquid and/or ice) water also contributes to the water vapor Raman echo, yielding unreasonable measurement results (e.g., relative humidity $>100 \%$ ). Over the years, there have been two different approaches to measure simultaneously vaporous and condensed water. One approach utilizes two discrete detection channels to extract the backscattered Raman signals respectively from condensed water and water vapor. When the lidar emits an ultraviolet radiation (e.g., $355 \mathrm{~nm}$ ), atmospheric (biogenic) aerosol-induced fluorescence or possible innerinstrument fluorescence might span a rather wide spectrum range which crams the entire Raman band of three-phase water. In this case, the discrete-channel Raman lidar would often produce false measurement results. Another approach introduces a spectrometer to record the full Raman spectra of water [1-4]. To convince the presence of (aerosol) fluorescence, Reichardt [4] proposed using spectral signals between 392 and $395 \mathrm{~nm}$ as an indicator, as no Raman scattering is observed in this spectrum area. However, the selected spectra are too narrow to effectively determine the fluorescence spectra, making it difficult to remove the fluorescence contamination from the Raman signals of water. Based on our former work [3], we present here a spectrallyresolved Raman lidar with well-arranged spectra coverage so that it can simultaneously record backscattered Raman spectrum signals from threephase water as well as aerosol fluorescence in the atmosphere. A spectra decomposition method is also developed to retrieve fluorescence spectra, water vapor Raman spectra and condensed (liquid and/or ice) water Raman spectra successively. Typical measurement results are provided to verify the lidar performance.

\section{LIDAR SETUP}

Fig. 1 shows a schematic layout of the spectrallyresolved Raman lidar. Compared to the former version [3], the main changes are summarized as following: (1) a beam splitter (BS) is placed after the first bandpass filter (BP1) to reflect $\sim 10 \%$ of incident light to be filtered as a reference $\mathrm{N}_{2}$ Raman signal. The BP1 has $>94 \%$ transmission in 


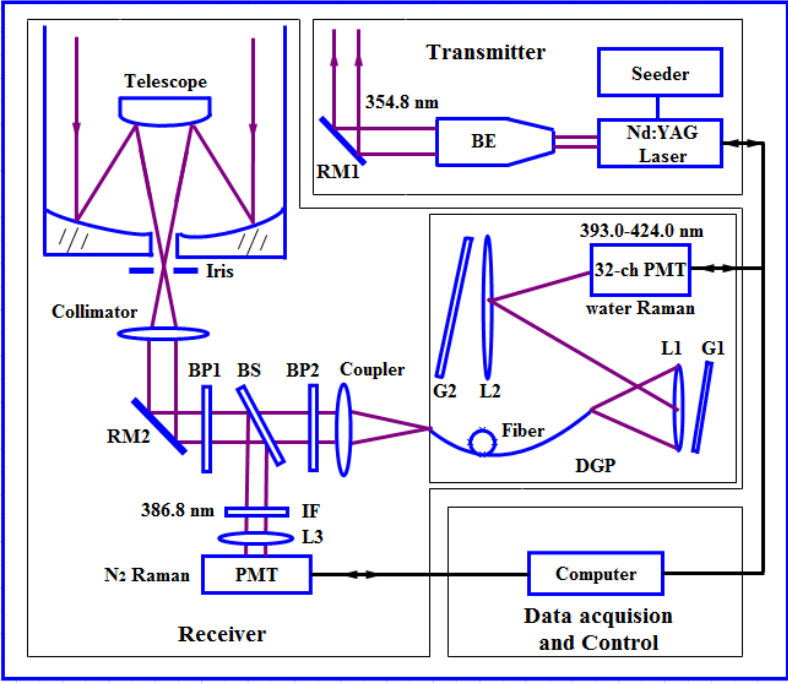

Fig. 1 Schematic layout of the spectrally-resolved Raman lidar. BE, beam expander; BP, bandpass filter; RM, reflecting mirror; $B S$, beam splitter; $I F$, interference filter; L, lens; $G$, grating; PMT, photomultiplier tub.

the 387-447 $\mathrm{nm}$ range and a suppression of $>6$ orders of magnitude to elastic signal around 354.8 $\mathrm{nm}$; (2) a second bandpass filter (BP2) is added before the fiber coupler to transmit $>80 \%$ of signal light in the $393.0-424.0 \mathrm{~nm}$ range, but reject elastic signal around $354.8 \mathrm{~nm}$ as well as the $\mathrm{O}_{2}$ and $\mathrm{N}_{2}$ Raman signals around 375.5 and $386.8 \mathrm{~nm}$ respectively with a suppression of more than 3 orders of magnitude; (3) the DGP (dualgrating polychromator) is updated to have an overall linear dispersion of $1.0 \mathrm{~mm} \mathrm{~nm}^{-1}$ in the 393-424 $\mathrm{nm}$ wavelength range (fiber: core diameter $=0.6 \mathrm{~mm}$, numerical aperture $=0.12 ; \mathrm{L} 1$ : diameter $=100 \mathrm{~mm}$, focal length $=300 \mathrm{~mm} ; \mathrm{G} 1$ : groove density $=600 \mathrm{gr} \mathrm{mm}^{-1}$, blazing angle $=6.89^{\circ}$, working angle $=9.27^{\circ}$; L2: diameter $=100 \mathrm{~mm}$, focal length $=400 \mathrm{~mm} ; \mathrm{G} 2$ : groove density $=600 \mathrm{gr}$ $\mathrm{mm}^{-1}$, blazing angle $=21.10^{\circ}$, working angle $=$ $21.72^{\circ}$ ). A 32-channel linear-array photomultiplier tubes (H7260-200, Licel, DE) is placed on the focal plane of the DGP. The spectrally-resolved Raman lidar records simultaneously the spectral signal in the 393-424 nm wavelength range with a $1.0-\mathrm{nm}$ resolution. For further understanding of the lidar system can refer to [3].

\section{SPECTRA DECOMPOSITION METHOD}

The lidar equation for each detection channel can be written as (background subtracted):

$$
\left\{\begin{array}{l}
N_{k}(z)=\frac{C_{k} \cdot O_{k}(z)}{z^{2}} \cdot \beta_{k, t}(z) \cdot \tau\left(\lambda_{0}\right) \cdot \tau\left(\lambda_{k}\right) \\
\beta_{k, t}=\beta_{k, F}+\beta_{k, V}+\beta_{k, L}+\beta_{k, I} \\
\lambda_{k}=(k-16)+407.57 \approx k+392 \mathrm{~nm} \\
k=1,2, \ldots 32
\end{array}\right.
$$

Here $N_{k}(z)$ denotes recorded photon counts at height $z$ for the $k^{\text {th }}$ detection channel, $C$ the system constant, $O$ the overlap function (become unity when $z>1 \mathrm{~km}$ ), $\tau$ the atmospheric transmission, $\lambda_{0}$ the laser wavelength and $\lambda_{k}$ the signal wavelength. The total $(t)$ volume backscatter coefficient $\beta$ is contributed by that of vaporous $(V)$, liquid $(L)$ and ice $(I)$ water plus fluorescent $(F)$ aerosol.

Defining normalized spectra $\left\{S_{k}\right\}$ :

$$
S_{k}=\frac{N_{k}}{N_{16}}=\frac{C_{k} \cdot O_{k} \cdot \tau\left(\lambda_{k}\right)}{C_{16} \cdot O_{16} \cdot \tau\left(\lambda_{16}\right)} \cdot \frac{\beta_{k, t}}{\beta_{16, t}}
$$

Considering the maximum wavelength deviation between $\lambda_{k}$ and $\lambda_{16}$ is $<17 \mathrm{~nm}$, the return-trip atmospheric transmission difference can well be neglected. As for the system overlap function $O_{k}$, they can be regarded as nearly identical to $O_{16}$ due to the optics of the DGP. Moreover, the ratios of $C_{k}$ to $C_{16}$ is calibrated by a white light source with known spectral characteristics (similar to [3]) and found to have values close to 1 . Thus for simplification here, the normalized spectra are approximated by:

$$
S_{k} \approx \frac{\beta_{k, F}+\beta_{k, V}+\beta_{k, L}+\beta_{k, I}}{\beta_{16, t}}
$$

For a 354.8-nm laser radiation, the water vapor, bulk water and ice generate vi-rotational Raman spectra in the spectrum ranges of 401-418 nm, $396-410 \mathrm{~nm}$ and 395-409 $\mathrm{nm}$, respectively. The spectral signals in the ranges of 393-394 and 419$424 \mathrm{~nm}$ are regarded as (aerosol) fluorescence, yielding normalized fluorescence spectra:

$$
S_{k}^{F} \approx \frac{\beta_{k, F}}{\beta_{16, t}}, k=1,2,27,28, \ldots 32
$$

Since the lidar has a limited wavelength detection range of only $32 \mathrm{~nm}$, an assumption is made that the fluorescence spectra vary slowly in the 393$424 \mathrm{~nm}$ range. Then the full fluorescence spectra 
in the 393-424 $\mathrm{nm}$ spectrum range are estimated (interpolated) using a linear fitting method:

$$
\left\{\begin{array}{l}
\left(\lambda_{k}, S_{k}^{F}\right), k=1,2,27,28, \ldots 32 \\
\quad \Downarrow \text { linear fitting } \\
\left\{S_{k}^{F}\right\}, k=1,2, \ldots 32
\end{array}\right.
$$

Simultaneous Equations (3) and (5), we get the normalized three-phase water Raman spectra in the 395-418 $\mathrm{nm}$ range:

$$
S_{k}^{w}=S_{k}-S_{k}^{F}=S_{k}^{V}+S_{k}^{L}+S_{k}^{I}, k=3,4, \ldots 26
$$

Under clear-sky conditions no cloud liquid and/or ice water are present, so Equation (6) regresses to:

$$
S_{k}^{w}=S_{k}-S_{k}^{F}=S_{k}^{V}, k=3,4, \ldots 26
$$

Equation (7) indicates that the normalized water vapor Raman spectra can be obtained under clearsky conditions.

After the retrieval of fluorescence spectra and water vapor Raman spectra, the condensed liquid and/or ice water Raman spectra in the 395-410 nm range are obtained:

$$
S_{k}^{L, I}=S_{k}-S_{k}^{F}-S_{k}^{V}, k=3,4, \ldots 18
$$

Thus, a spectra decomposition method has been developed to retrieve fluorescence spectra, water vapor Raman spectra and condensed water Raman spectra successively. The liquid and ice water Raman spectra can also be separated, but to economize words, we don't make any further discussion here. Moreover, the separated true water vapor Raman signals, e.g.:

$$
N_{16}^{V}=N_{16} \cdot S_{16}^{V}
$$

can be directly used to retrieve the water vapor content. Similarly, the liquid and/or ice water content in cloud area can also be determined.

\section{TIPICAL MEASUREMENT RESULTS}

\subsection{Clear-sky case}

Fig. 2 presents measurement result performed on the night of July 28,2015 . The weather was clear as a co-located polarization lidar convinced that there were no clouds or obvious aerosol load present in laser sight. The data between 21:00 and 22:00 LT and in the height range of 1.5-2.0 km were accumulated to generate the normalized spectra (Fig. 2a). The spectra only show a single peak around $407.57 \mathrm{~nm}$, corresponding to the Qbranch Raman spectra of water vapor. The fluorescence spectra are first separated (Fig. 2b, magenta) using the method introduced above. The residual spectra (Fig. 2b, blue) are regarded as lidar-measured water vapor Raman spectra.

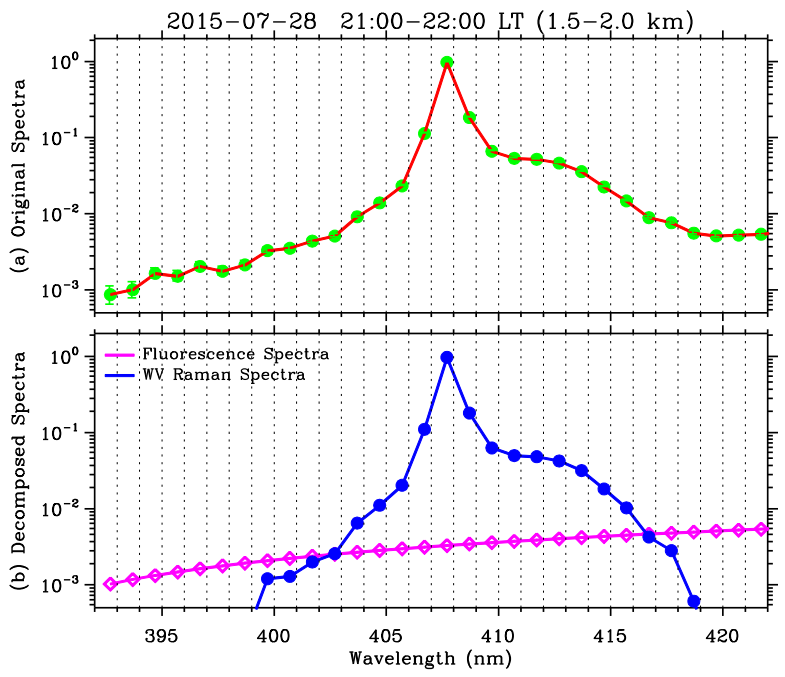

Fig. 2 (a) original normalized spectra by the spectrally-resolved Raman lidar. Error bars indicate statistical uncertainty; (b) separated spectral components after applying the spectra decomposition method: fluorescence spectra (magenta) and lidar-measured water vapor Raman spectra (blue).

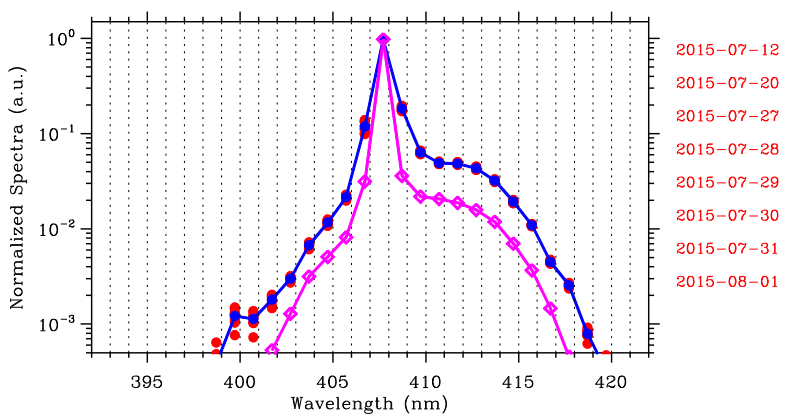

Fig. 3 Lidar-measured water vapor Raman spectra: red, nightly-mean spectra for 8 different clear-sky nights; blue, their average spectra; magenta, theoretical water vapor Raman spectra (according to [5]) smoothed with a 1.0-nm resolution. Note the lidar-measured water vapor Raman spectra are broadened by the lidar receiving optics, but nearly invariant and quite similar to the theoretical spectra in shape. 
Fig. 3 shows the results of lidar-measured water vapor Raman spectra on 8 different clear-sky nights. For each night, water vapor Raman spectra from different 1-h integration periods are retrieved and averaged to yield nightly-mean spectra (red). The blue curve plots the average spectra of the 8 nights. For comparison, the theoretical water vapor Raman spectra calculated according to [5] and smoothed with a 1.0-nm resolution (Fig. 3, magenta) are also added. It can be seen that the lidar-measured spectra are broadened by the lidar receiving optics, but quite similar in shape to the theoretical spectra. Note the lidar-measured water vapor Raman spectra are nearly invariant, thus can serve as background reference for separation of mixed-phased water Raman spectra.

\subsection{An ice virga case}

The co-located and concurrent polarization lidar indicated that there were clouds in the height range of 2.5-3.5 km and between 03:00 and 04:00 LT on the night of Jan 01, 2016 with volume depolarization ratio values of $>0.3$ (Figures not provided here). It is regarded as an ice virga event. Fig. 4a shows the original normalized spectra obtained by the spectrally-resolved Raman lidar and a second peak is visible near $400 \mathrm{~nm}$. After applying the above spectra decomposition method, Fig. $4 \mathrm{~b}$ plots the successively separated spectra components: fluorescence spectra (magenta), total mixed-phased water Raman spectra (dash black), vaporous (solid blue) and condensed (solid red) water Raman spectra. For comparison, theoretical water vapor (dash blue) and ice (dash red) water Raman spectra are also added. The theoretical ice Raman spectra data are taken from Slusher and Derr (1975). It is found the extracted condensed water Raman spectra are highly similar to the theoretical ice water Raman spectra, convincing that the cloud is dominated by ice water which is consistent with the polarization lidar measurement results. The cloud ice water content can further be determined using the separated ice water Raman signals.

In conclusion, the presented spectrally-resolved Raman lidar is capable of measuring atmospheric three-phase water simultaneously despite presence of fluorescent aerosols. It's regarded as an effective tool for studying of cloud microphysics and interaction between aerosols and clouds.

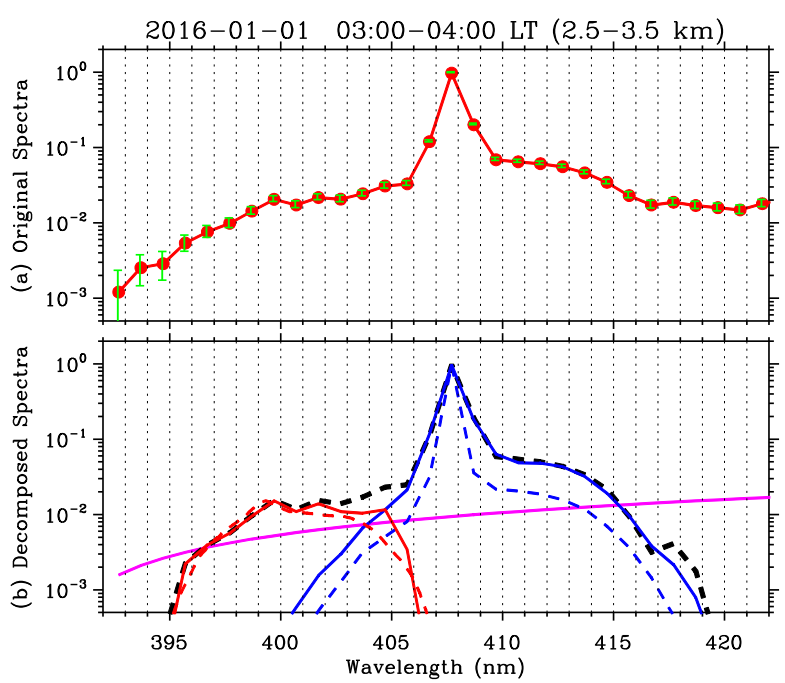

Fig. 4 Spectra measurement results for an ice virga event: (a) original normalized spectra with a second peak near $400 \mathrm{~nm}$; (b) separated spectra components: fluorescence spectra (magenta), total mixed-phased water Raman spectra (dash black), vaporous (solid blue) and condensed (solid red) water Raman spectra. Theoretical water vapor (dash blue) and ice (dash red) water Raman spectra are added for comparison. The theoretical ice Raman spectra are taken from [6].

\section{ACKNOWLEDGEMENTS}

This work was supported by the National Natural Science Foundation of China (No. 61505148) and National Key Scientific Instrument and Equipment Development Project (No. 41327801).

\section{REFERENCES}

[1] D. Kim, et al. Lidar measurement of a full Raman spectrum of water by using a multichannel detector, $J$. Korean Phys. Soc., 54, 38-43 (2009) .

[2] T. Sakai, et al. Liquid water cloud measurements using the Raman lidar technique: Current understandding and future research needs, J. Atmos. Oceanic Technol., 30, 1337-1353 (2013) .

[3] F. Liu and F. Yi. Spectrally resolved Raman lidar measurements of gaseous and liquid water in the atmosphere, Appl. Opt., 52(28), 6884-6895 (2013) .

[4] J. Reichardt. Cloud and Aerosol Spectroscopy with Raman Lidar, J. Atmos. Oceanic Technol., 31, 19461963 (2014).

[5] G. Avila, et al. Ro-vibrational Raman cross sections of water vapor in the $\mathrm{OH}$ stretching region, J. Mol. Spectrosc., 196, 77-92 (1999).

[6] R. B. Slusher, V. E. Derr. Temperature dependence and cross sections of some Stokes and anti-Stokes lines in ice Ih, Appl. Opt., 14, 2116-2120 (1975). 